\title{
An Easily Neglected Side During Bromhidrosis Surgery?
}

Wen-Tsao Ho ${ }^{1}$, Jiun-Yit Pan ${ }^{2}$, Miao-Erh Chang ${ }^{1}$ and Yi-Jen Peng ${ }^{3^{*}}$

${ }^{1}$ Department of Dermatology, Ho Wen Tsao skin clinic, New Taipei city, Taiwan

${ }^{2}$ Department of dermatology, National Skin Centre, 1 Mandalay Road, Singapore

${ }^{3}$ Department of Pathology, Tri-Service General Hospital, National Defense Medical Center, Taipei, Taiwan

The bromhidrosis surgery is an effective and efficient way to get rid of body malodour by eradicating the apocrine glands [1]. This procedure might cause negative side effects, such as hematoma, skin flap necrosis, scar formation, or odour recurrence [2]. To achieve more satisfactory outcomes, surgeons should try to avoid these complications during surgery [3]. Studies have shown that incomplete apocrine gland removal may be the primary cause of odour recurrence. Consequently, surgeons now focus on completely removing the apocrine glands from the axillary skin flaps during surgery [4]. Such removal is very important because apocrine is part of a subcutaneous follicular unit, and it contributes to the aetiology of body malodour.

When bromhidrosis surgery is performed, whether via liposuctionassisted curettage or minimally invasive surgery, a tumescent solution is first delivered between the skin and subcutaneous tissue, and iris scissors are used to separate them. The apocrine glands are subsequently removed carefully from the skin flaps. Interestingly we have observed high recurrences in our patients, such as with previous liposuction for accessory breast or trans-axillary breast augmentation, although these kinds of patients only occupied few in our bromhidrosis surgeries. We are curious about it and suspect the apocrine glands and superficial fascia complex are destroyed and not intact on the side of the skin flap after iris scissor dissection. This is why some of the glands are instead separated and attached toward the side of the subcutaneous tissues.

\author{
Publication History: \\ Received: July 12, 2016 \\ Accepted: November 29, 2016 \\ Published: December 01, 2016
}

\section{Keywords:}

Bromhidrosis Surgery, Invasive surgery, apocrine glands

We could deduce that the previous axillary surgery may destroy the superficial fascia complex and make the plane disoriented. The apocrine glands not easily seen on the side of subcutaneous tissue after dissection is found abundant on the ones who had undergone axillary surgery before. Although many surgeons are aware of the importance of clearing the apocrine glands from the skin flap to reduce the risk of malodour recurrence, glands located in the subcutaneous tissue are easily neglected in patients who have previously undergone axillary surgery and these glands may also make a minor contribution to recurrence. Therefore, surgeons must consider the presence of apocrine glands both on the skin flap and on the subcutaneous adipose in order to achieve the best outcomes. However, we do not recommend that operators take an unnecessary amount of tissue, as this might increase the risk of hematoma and subsequent skin necrosis. Instead, we suggest that they remove the suspected glands in the subcutaneous adipose after careful inspection. We presented this interesting case and would like to arouse attentions for further investigations for these conditions.
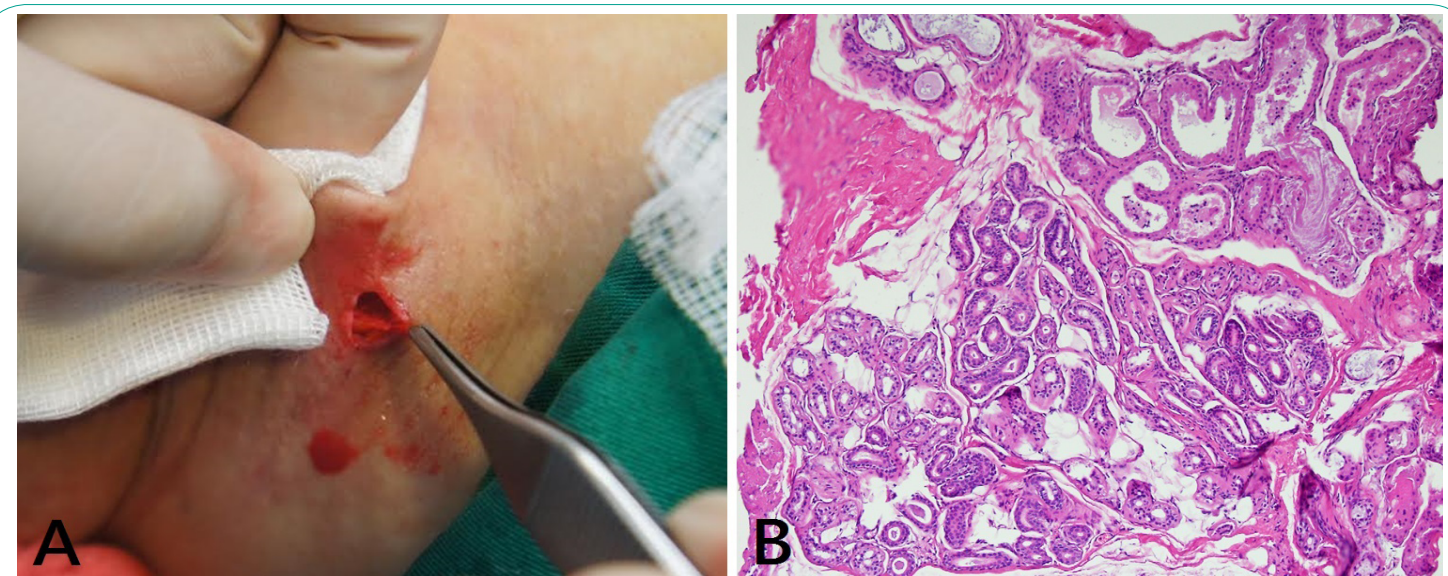

Figure 1: (A) Some tissues on the subcutaneous fat were of pink color instead of the normal pure yellowish fat. (B Microscopically, the apocrine glands were found to be intermingled with some fibrotic tissue and fat cells $\times 200$.

We present the case of a 40 year-old patient who had undergone trans-axillary breast augmentation 10 years prior to undergoing bromhidrosis surgery in our department. During bromhidrosis surgery, we noted that some of the subcutaneous adipose tissues were pink instead of the normal yellowish colour of pure adipose (Figure 1a). The suspicious tissues were excised and sent for pathological examination. Under microscopic examination, apocrine glands were found to be intermingled with fibrotic tissue and adipocytes (Figure 1b). Based on this evidence, we thought that the body malodour could recur if the glands were not thoroughly removed from both the skin and the adipose tissue.
"Corresponding Author: Dr. Yi-Jen Peng, Department of Pathology, Tri-Service General Hospital, National Defense Medical Center, Taipei, Taiwan; E-mail: yijen0426@gmail.com

Citation: Ho WT, Pan JY, Chang ME, Peng YJ (2016) An easily Neglected Side During Bromhidrosis Surgery? Int J Surg Surgical Porced 1: 111. doi: http:// dx.doi.org/10.15344/ijssp/2016/111

Copyright: @ $2016 \mathrm{Ho}$ et al. This is an open-access article distributed under the terms of the Creative Commons Attribution License, which permits unrestricted use, distribution, and reproduction in any medium, provided the original author and source are credited. 
Citation: Ho WT, Pan JY, Chang ME, Peng YJ (2016) An easily Neglected Side During Bromhidrosis Surgery? Int J Surg Surgical Porced 1: 111. doi: http://dx.doi. org/10.15344/ijssp/2016/111

Page 2 of 2

\section{Competing Interests}

The authors declare that they have no competing interests.

\section{References}

1. Mao GY, Yang SL, Zheng JH (2008) Etiology and management of axillary bromidrosis: a brief review. Int J Dermatol 47: 1063-1068.

2. Seo SH, Jang BS, Oh CK, Kwon KS, Kim MB (2008) Tumescent superficial liposuction with curettage for treatment of axillary bromhidrosis. J Eur Acad Dermatol Venereol 22: 30-35.

3. He J, Wang T, Dong J (2012) Excision of apocrine glands and axillary superficial fascia as a single entity for the treatment of axillary bromhidrosis. J Eur Acad Dermatol Venereol 26: 704-709.

4. Wang R, Yang J, Sun J (20115) A Minimally Invasive Procedure for Axillary Osmidrosis: Subcutaneous Curettage Combined with Trimming Through a Small Incision. Aesthetic Plast Surg 39:106-113. 